\title{
Direct Observation of Hydrogen in Cold-Drawn Pearlitic Steel Wires Using Cryogenic Atom Probe Tomography
}

\author{
Andrew J Breen ${ }^{1 *}$, Yujiao Li $^{2}$, Leigh Stephenson ${ }^{1}$, Baptiste Gault ${ }^{1,3}$ and Michael Herbig ${ }^{1}$ \\ 1. Max-Planck-Institut für Eisenforschung GmbH, Department of Microstructure Physics and \\ Alloy Design (MA), Düsseldorf, Germany \\ 2. Center for Interface-Dominated High Performance Materials (ZGH), Ruhr-University \\ Bochum, Bochum, Germany \\ 3. Imperial College, Royal School of Mines, Department of Materials, London, SW7 2AZ, \\ UK \\ *Corresponding author: a.breen@mpie.de
}

Hydrogen embrittlement (HE) remains a formidable challenge in many high strength alloys. The ingress of hydrogen $(\mathrm{H})$ can lead to a sudden loss of ductility and premature failure of the alloy. The mechanisms responsible for $\mathrm{HE}$ are almost entirely understood through a combination of theoretical modeling and indirect experimental observations. Direct atomic scale observations of $\mathrm{H}$ are lacking and promise to elucidate key processes responsible for $\mathrm{HE}$.

Atom probe tomography (APT), unique in its ability to reconstruct the position and chemical identity of millions of individual atoms in 3D from a material specimen, is currently the only technique that has the potential to directly locate the 3D distribution of $\mathrm{H}$ atoms at the atomic scale. However, quantification of $\mathrm{H}$ using APT remains challenging, due in part to the residual $\mathrm{H}$ always present in the analysis chamber, as well the mobile nature of the $\mathrm{H}$ being analyzed. To mitigate these challenges, ambitious experimental protocols involving deuterium (D) charging and UHV cryogenic sample transfer [1] are being developed. Successful detection and microstructural characterization of stable hydrides and deuterides using APT have recently been reported [2, 3]. H/D at carbide trapping sites in steel have also been shown [4, 5]. However, what remains is the characterization of solute hydrogen at other microstructural features, such as grain boundaries, where much of the damage responsible for HE is likely to be occurring.

Heavily cold-drawn pearlitic steel wires with hypereutectoid composition (Fe-0.98C-0.31Mn$0.20 \mathrm{Si}-0.20 \mathrm{Cr}-0.01 \mathrm{Cu}-0.006 \mathrm{P}-0.007 \mathrm{~S}$ wt.\%) provide an interesting case study. Recently reported to achieve tensile strengths of over $5 \mathrm{GPa}$ [6], they are among the strongest structural bulk alloys known, but nevertheless, they are susceptible to HE. A series of experiments was designed to compare the content and atomic distribution of $\mathrm{H} / \mathrm{D}$ in the wires in the as-received condition, after in-situ low temperature annealing for 4 hours at $150{ }^{\circ} \mathrm{C}$ in vacuum and after electrochemical deuterium charging with and without cryogenic transfer. Samples were electrolytically sharpened and electrochemical charging was carried out inside a $\mathrm{N}_{2}$ filled glovebox using a potentiostat and charging cell (-1.2 V using a $0.1 \mathrm{M} \mathrm{NaOD}$ in $\mathrm{D}_{2} \mathrm{O}$ solution). All specimens were run on a CAMECA $^{\circledR}$ LEAP $^{\circledR} 5000 \mathrm{XR}$ at $60 \mathrm{~K}$ and $15 \%$ pulse fraction voltage pulsing. A Ferrovac ${ }^{\circledR}$ cryogenic UHV suitcase was used to transfer specimens directly to the atom probe after deuterium charging for the final experimental workflow at pressures on the order of $10^{-9}$ mbar and a temperature of approximately $-180{ }^{\circ} \mathrm{C}$ in an effort to reduce the amount of charged D egressing from the specimens before the APT experiments (Fig.1).

A significant change in the detected $\mathrm{H} / \mathrm{D}$ levels after the different experimental workflows was observed (Fig. 2). In the as-received condition, 0.08 at.\% of $\mathrm{H}$ was measured. After annealing, only 0.02 at. $\%$ of $\mathrm{H}$ was measured - a dramatic reduction of $75 \%$. It follows that the majority 
of $\mathrm{H}$ measured in the as-received condition was from the specimen and not from the analysis chamber. After annealing, the remaining $\mathrm{H}$ can therefore be assumed to be comprised of residual $\mathrm{H}$ in the analysis chamber and $\mathrm{H}$ contained in deep trapping sites of the steel. Interestingly, after deuterium charging without cryogenic transfer, D was still detected at the interfaces between ferrite and decomposed cementite, even after 24 days after charging. After D charging and cryogenic transfer, strong D signal was observed in the decomposed cementite regions. The results provide information of the H/D content and distribution within ultra-high strength pearlitic steel wires and important insight into the mechanisms of HE [7].

\section{References:}

[1] LT Stephenson et al., Plos one 13 (2018), p. e0209211

[2] AJ Breen et al., Scripta Materialia 156 (2018), p. 42.

[3] Y Chang et al., Acta Materialia 150 (2018), p. 273.

[4] Y-S Chen et al., Science 355 (2017), p. 1196.

[5] J Takahashi, K Kawakami and Y Kobayashi, Acta Materialia 153 (2018), p. 193.

[6] Y Li et al., Physical Review Letters 113 (2014), p. 106104.

[7] The authors wish to thank U Tezins, A Sturm, I Mouton, F Yan and Y Chang for support at MPIE. AJ Breen acknowledges the Alexander von Humboldt $(\mathrm{AvH})$ foundation for funding through the award of a postdoctoral fellowship.
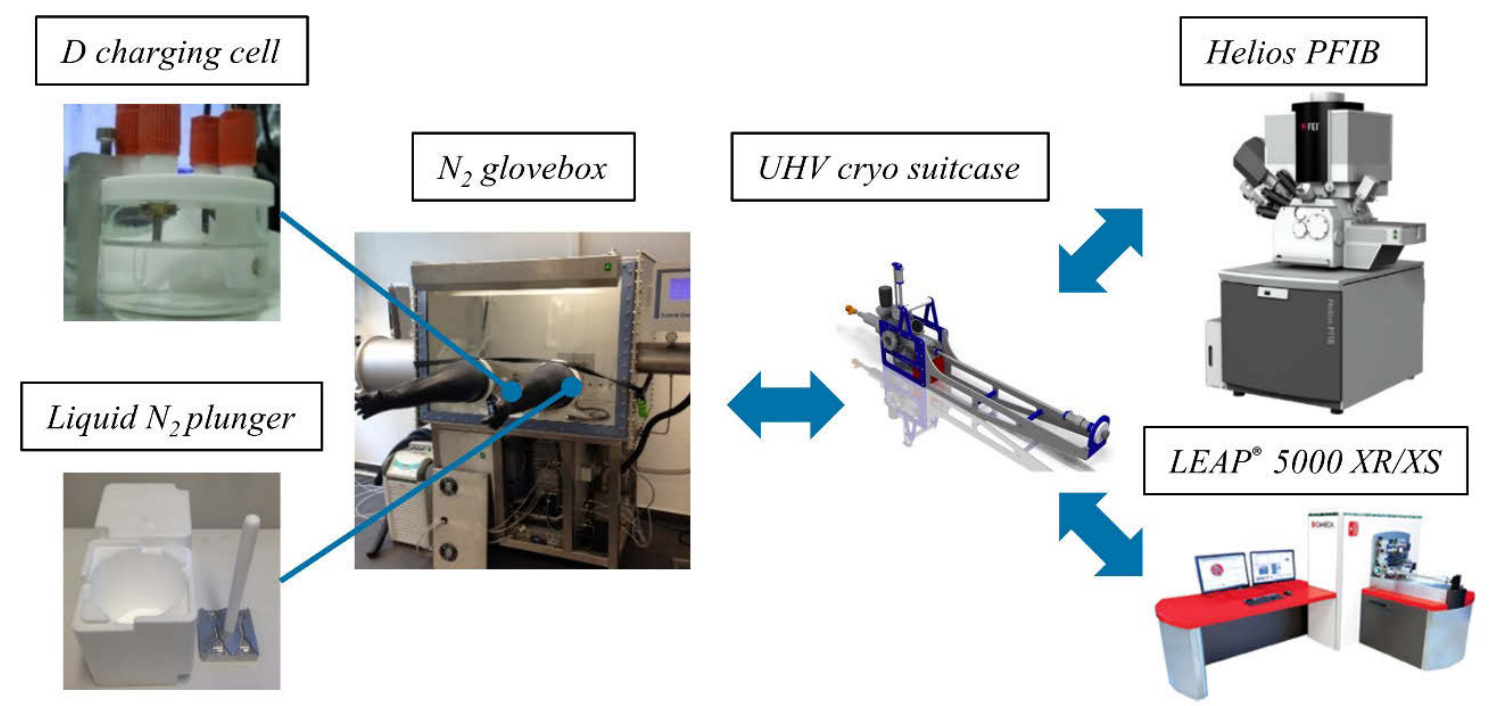

Figure 1. Experimental overview: Deuterium charging and plunge freezing are done inside an $\mathrm{N}_{2}$ filled glovebox. Samples are then transferred using a UHV cryogenically cooled suitcase to either the PFIB for imaging and additional sharpening or directly to the LEAP ${ }^{\circledR} 5000 \mathrm{XR} / \mathrm{XS}$.

(a)

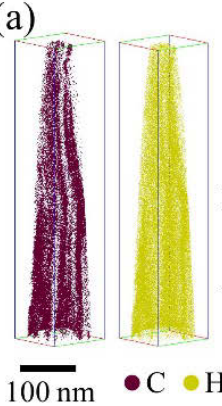

(b)

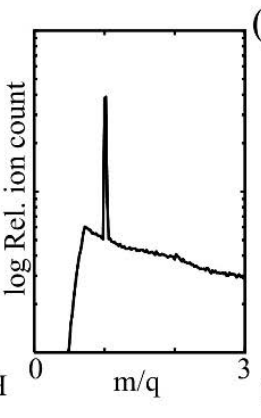

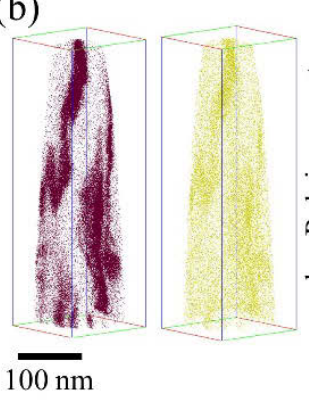

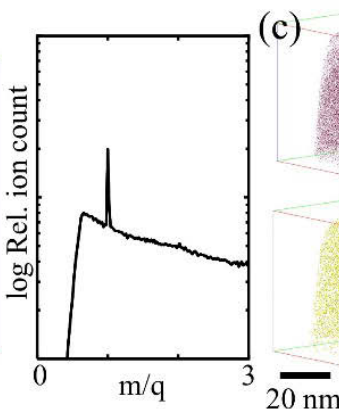

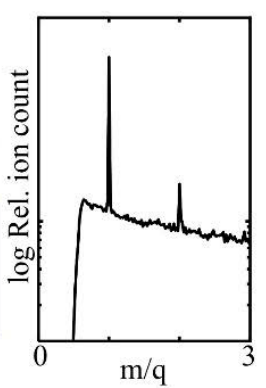

Figure 2. APT reconstructions and H/D mass spectra in (a) as received, (b) after low temperature annealing @ $150^{\circ} \mathrm{C}$ in vacuum and (c) after deuterium charging + cryo transfer. 